\title{
Measurement of Nanometer Electron Beam Sizes Using Laser Interference by Shintake Monitor
}

\author{
${\text { Jacqueline } \text { Yan }^{\mathrm{a}}{ }^{*}, \text { Yoshio Kamiya }^{\mathrm{b}} \text {, Sachio Komamiya }}^{\mathrm{a}}$, \\ Toshiyuki Okugi ${ }^{\mathrm{c}}$, Nobuhiro Terunuma ${ }^{\mathrm{c}}$, Kiyoshi Kubo ${ }^{\mathrm{c}}$, Toshiaki Tauchi ${ }^{\mathrm{d}}$, \\ ${ }^{a}$ Department of Physics, The University of Tokyo, Tokyo 113-0033, Japan \\ ${ }^{b}$ International Center for Elementary Particle Physics, The University of Tokyo, Tokyo 113-0033, Japan \\ ${ }^{c}$ Accelerator Laboratory, KEK, Ibaraki 305-0801, Japan \\ ${ }^{\mathrm{d}}$ Institute of Particle and Nuclear Studies, KEK, Ibaraki 305-0801, Japan
}

\begin{abstract}
The Shintake Monitor is an essential beam tuning device installed at the interaction point of ATF2, the final focus test beam line for ILC, to measure its $\mathrm{O}(10) \mathrm{nm}$ order vertical $\mathrm{e}^{-}$beam sizes $\left(\sigma_{\mathrm{y}}\right)$. The $\mathrm{e}^{-}$ beam collides with a target of laser interference fringes, and $\sigma_{\mathrm{y}}$ is derived from the modulation depth of the resulting Compton signal photons measured by a downstream photon detector. The laser optics is designed to accommodate a wide range of $\sigma_{\mathrm{y}}$ from $25 \mathrm{~nm}$ to a few $\mu \mathrm{m}$ with better than $10 \%$ accuracy. Being the only existing device capable of measuring $\sigma_{\mathrm{y}}<100 \mathrm{~nm}$, the Shintake Monitor is crucial for verifying ATF2's Goal 1 of focusing $\sigma_{\mathrm{y}}$ down to the design value of $37 \mathrm{~nm}$. It has demonstrated measurement stability of about 5\%. Major improvements in hardware and measurement schemes contributed to suppressing error sources. This paper describes the design concepts and performance of Shintake Monitor, focusing on an extensive study of systematic errors with the aim of precisely extracting $\sigma_{\mathrm{y}}$ from the measured modulation.
\end{abstract}

Technology and Instrumentation in Particle Physics 2014

2-6 June, 2014

Amsterdam, the Netherlands

Mail to : jackie@icepp.s.u-tokyo.ac.jp

\section{Introduction}

The International Linear Collider (ILC) design[1] demands a high luminosity of $1.8 \times 10^{34} \mathrm{~cm}^{-2} \mathrm{~s}^{-1}$ for the detailed research of the Higgs boson and new physics beyond the Standard Model. This requires the beam sizes at the interaction point (IP) to be focused to $\left(\sigma_{x}, \sigma_{y}\right)=(474,5.9) \mathrm{nm}$. At ATF2 (KEK), the test prototype of the ILC final focus system, the Local Chromaticity Correction scheme[2] has been demonstrated by focusing and stable measurement of $\sigma_{\mathrm{y}}$ to below $100 \mathrm{~nm}$. ATF2's "Goal 1" is to ultimately focus $\sigma_{y}$ to $37 \mathrm{~nm}$, the design size energy-scaled down from ILC's beam energy of $250 \mathrm{GeV}$ to ATF's of $1.3 \mathrm{GeV}$ [3]. "Goal 2" is nm-level beam position stabilization at the IP. The Shintake Monitor [4][5], installed at ATF2's IP, is the only existing device capable of measuring such a miniscule $\sigma_{\mathrm{y}}$. Its outcomes are indispensable for achieving "Goal 1", and thus for demonstrating the feasibility of realizing the ILC.

\section{The Shintake Monitor System}

\subsection{Layout and Measurement Scheme of the Shintake Monitor}

Figure 1 shows the schematic layout of the Shintake Monitor system, which consists of laser optics and a gamma detector[7][8]. The laser source located outside the accelerator tunnel generates Nd:YAG Q-switch laser pulses of wavelength $532 \mathrm{~nm}$ by second harmonic generation. The pulse energy is $0.56 \mathrm{~J}$ and the FWHM pulse width is $8 \mathrm{~ns}$. The laser is delivered through a $20 \mathrm{~m}$ transport system containing lenses to adjust the spot size and divergence, to an upright standing optical table

${ }^{1}$ Shintake Monitor, invented by Dr. T. Shintake, was first put into practical usage at the FFTB experiment at SLAC [6]. 
at the IP, then split into upper and lower paths by a $50 \%$ beam splitter. The two paths are each focused by lenses to a tight waist at the IP, where they cross to form the interference fringes that the $\mathrm{e}^{-}$beam collides perpendicularly against. The fringe phase is scanned relative to the $\mathrm{e}^{-}$beam by a piezoelectric stage, which produces Compton scattered photon signal with modulation (M) that is measured by a downstream gamma detector made up of layers of CsI(Tl) scintillators. After collision, the beam is bent by a dipole magnet safely into a dump. The smaller $\sigma_{y}$ is w.r.t. the fringe pitch, the larger the observed $\mathrm{M}$ will be (Fig. 2). The number of signal photons (N) is calculated by Eq. 1 as the convolution of a Gaussian beam distribution and the laser fringe intensity. Laser fringe intensity os expressed using the magnetic field in the laboratory frame.

$$
N \propto \int_{-\infty}^{\infty} \frac{1}{\sqrt{2 \pi} \sigma_{y}} \exp \left(-\frac{\left(y-y_{0}\right)^{2}}{2 \sigma_{y}^{2}}\right) \overline{\left(B_{x}^{2}+B_{y}^{2}\right)} d y \Rightarrow N=\frac{N_{0}}{2}\left\{1+\cos \left(2 k_{y} y_{0}\right) \cos \theta \exp \left(-2\left(k_{y} \sigma_{y}\right)^{2}\right)\right\}
$$

Here, $\mathrm{k}_{\mathrm{y}}=\mathrm{k} \sin (\theta / 2)$ is the vertical component of laser wave number $\mathrm{k}=2 \pi / \lambda(\theta$ : crossing angle, $\lambda$ : laser wavelength), and $\mathrm{y}_{0}$ is beam center position. $\mathrm{M}$, interpreted as the ratio of amplitude to average of signal intensity, is expressed as in Eq. 2 using its maximum $\left(\mathrm{N}_{+}\right)$and minimum $\left(\mathrm{N}_{-}\right)$. Then $\sigma_{\mathrm{y}}$ is calculated from the measured $\mathrm{M}$ as Eq. 3. Measurable range is determined by the fringe pitch " $\mathrm{d}$ ", which depends on $\theta$ (Table 1).

$$
\begin{aligned}
& M=\frac{N_{+}-N_{-}}{N_{+}+N_{-}}=|\cos \theta| \cdot \exp \left[-2\left(k_{y} \sigma_{y}\right)^{2}\right] \\
& \sigma_{y}=\frac{d}{2 \pi} \sqrt{2 \ln \left(\frac{\cos \theta \mid}{M}\right)} \quad d=\frac{\pi}{k_{y}}=\frac{\lambda}{2 \sin (\theta / 2)}
\end{aligned}
$$

Figure 3 relates $\mathrm{M}$ and $\sigma_{\mathrm{y}}$ for each $\theta$. The maximum $\mathrm{M}$ comes up to $\cos \theta$. For the larger $\sigma_{\mathrm{y}}, 2^{\circ}-8^{\circ}$ (continuously adjustable) is used. As $\sigma_{\mathrm{y}}$ focusing progresses, "mode switching" to $30^{\circ}$ and finally $174^{\circ}$ is necessary in order to maintain good measurement resolution. This is carried out by remote control of stages carrying mirror actuators.

Table 1: Measurable $\sigma_{\mathrm{y}}$ vary with fringe pitch, which depends on $\theta$.

\begin{tabular}{ccccc}
\hline Crossing angle $\boldsymbol{\theta}$ & $174^{\circ}$ & $30^{\circ}$ & $8^{\circ}$ & $2^{\circ}$ \\
\hline Fringe pitch d & $266 \mathrm{~nm}$ & $1.028 \mu \mathrm{m}$ & $3.81 \mu \mathrm{m}$ & $15.2 \mu \mathrm{m}$ \\
\hline Measurable $\boldsymbol{\sigma}_{\mathbf{y}}$ & $25-100 \mathrm{~nm}$ & $100-360 \mathrm{~nm}$ & $360 \mathrm{~nm}-1.4 \mu \mathrm{m}$ & $1.4--6.0 \mu \mathrm{m}$ \\
\hline
\end{tabular}

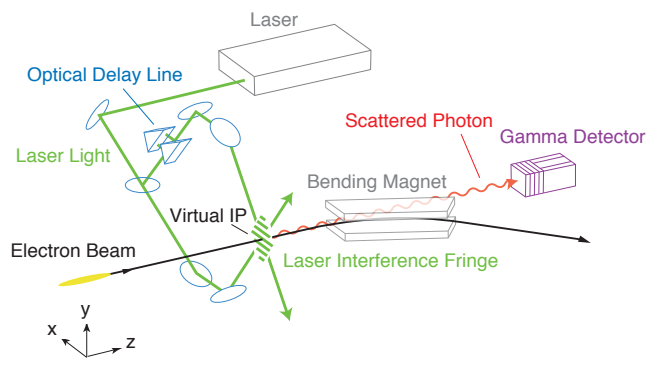

Fig 1: Schematic layout of Shintake Monitor [7]

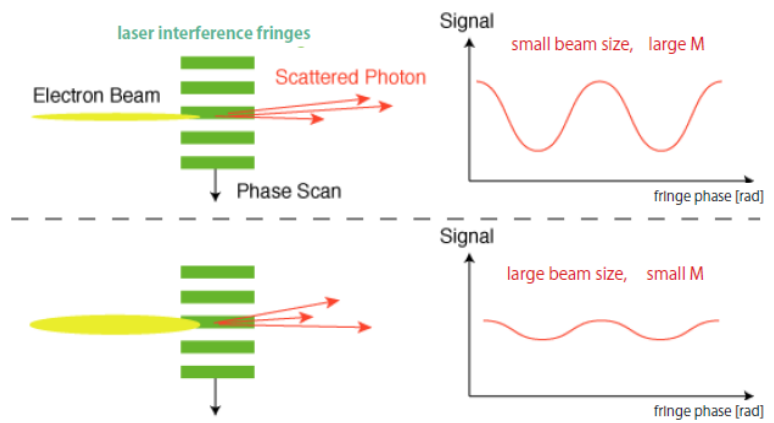

Fig 2. Relationship between $\sigma_{y}$ and modulation depth 


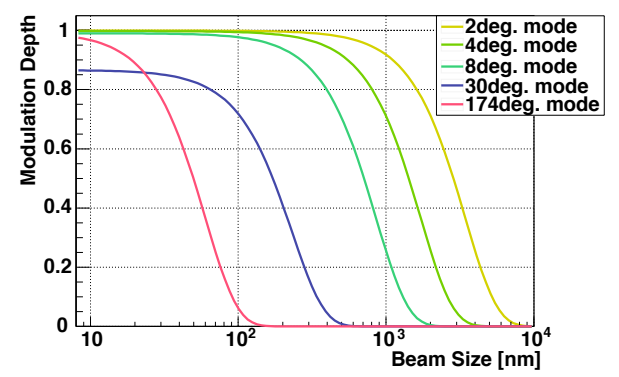

Fig 3: Plot of modulation depth vs $\sigma_{\mathrm{y}}$ for each $\theta$ mode[7]

\section{Performance}

\subsection{Procedure and status during beam time}

The Shintake Monitor contributes essentially to $\sigma_{\mathrm{y}}$ focusing at ATF2. Its usage commences after $\sigma_{\mathrm{y}}$ has been initially tuned to $<2 \mu \mathrm{m}$, confirmed by wire scanners. Detection of clear M requires the laser to be sufficiently focused by lenses and precisely aligned to the beam at the IP. Alignment is carried out in transverse and longitudinal directions by scanning mirror angles using actuators[9]. After these preparations, the results of $\sigma_{\mathrm{y}}$ measurements by fringe scans are fed back to the beam focusing process by sextupole magnet tuning knobs[10]. In practice, the measured Compton energy spectrum is plotted as a function of fringe phase $(\varphi)$, and fitted with the following cosine-like function:

$$
E_{\text {sig }}=E_{\text {avg }}\left[1+M \cdot \cos \left(\varphi+\varphi_{0}\right)\right] \quad\left(\varphi=2 k_{y} y\right)
$$

The parameters extracted from fitting are $\mathrm{M}, \mathrm{E}_{\text {avg }}$ (average energy), and $\varphi_{0}$ (initial phase). Fig. 4 (right) shows an example of a fringe scan.

By Mar, 2013, consistent measurements of $\sigma_{\mathrm{y}}<65 \mathrm{~nm}$ had been achieved[11]. In spring of 2014, various hardware improvements were carried out to reduce signal jitters and drifts and suppress systematic errors (see Sec 3.2). These include adjusting the laser profile to reduce pointing jitter at the IP, laser timing stabilization, e- beam orbit stabilization, and speeding up DAQ software to lighten effects from slow drifts. Owing to these efforts, relatively high $M$ was measured at $174^{\circ}$ mode in Apr-Jun of 2014, with measurement stability improved to as good at about $5 \%$. This enables very effective beam tuning. The beam size can be reduced from above $150 \mathrm{~nm}$ to below $60 \mathrm{~nm}$ in just half a day. In the example in Fig 4 (left), 10 scans at $174^{\circ}$ mode were conducted consecutively within about $30 \mathrm{~min}$. Without taking into account of systematic errors of Shintake Monitor, $\mathrm{M}_{\text {meas }}=0.341+/-0.025$ (stat.) corresponds to $\sigma_{\mathrm{y}, \text { meas }}=61.9 \pm 2.1 \mathrm{~nm}$ (stat.). For status of even smaller $\sigma_{\mathrm{y}}$ measured in 2014, refer to [12]. Furthermore, it has been demonstrated that long term stable performance is maintained under various conditions e.g. finer-step or prolonged ranges. The rest of this paper describes the ongoing study of $\mathrm{M}$ reduction factors, which works almost always to over-evaluate $\sigma_{\mathrm{y}}$. Shintake Monitor is also used for beam study of factors suspected to cast limitation on small $\sigma_{\mathrm{y}}$ focusing [11][12][13].
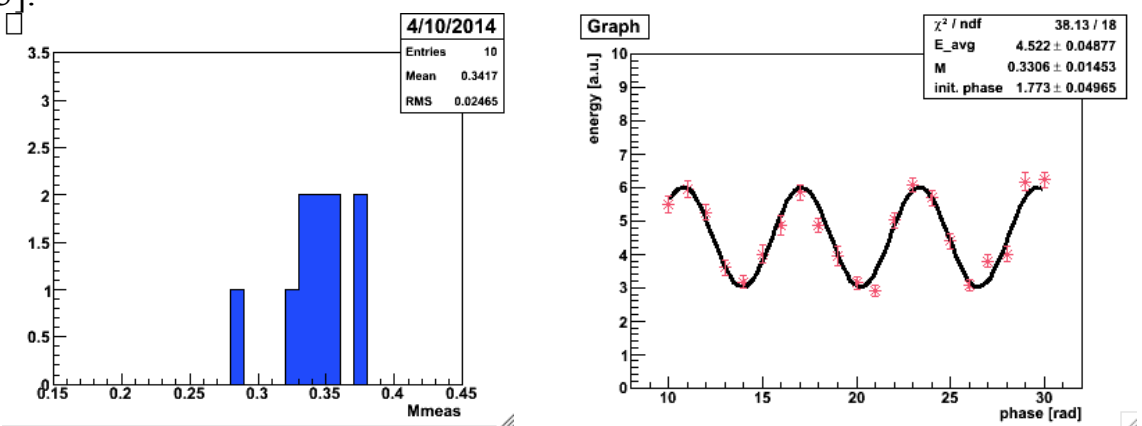

Fig 4: $\mathrm{M}$ measured by fringe scans in the $174^{\circ}$ mode. (left) : 10 continuous scans resulted in $\mathrm{M}_{\text {meas }}=0.341+/-0.025$ (stat.). Without taking into account of systematic errors of Shintake Monitor, this corresponds to $\sigma_{y, \text { meas }}==61.9 \pm 2.1 \mathrm{~nm}$. (stat.) (right) : one scan from this set: $\mathrm{M}_{\text {meas }} \sim 0.33, \sigma_{\mathrm{y} \text {,meas }} \sim 63 \mathrm{~nm}$. Plotted here are the average and S.D, of 10 pulses used for measurement at each phase. 


\subsection{Modulation Reduction Factors}

Systematic errors are interpreted using "modulation reduction factors". These smear fringe contrast and cause under-evaluation of the measured $\mathrm{M}$ as $\mathrm{M}_{\text {meas }}=\prod_{i} C_{i} \mathrm{M}_{\mathrm{ideal}}$. Here, $\mathrm{C}_{\mathrm{i}}$ represent individual $\mathrm{M}$ reduction factors. This leads to the beam size over-evaluation as

$$
\sigma_{y} \rightarrow \sqrt{\sigma_{y}^{2}+\sum_{i}\left|\ln \left(C_{i}\right)\right| /\left(2 k_{y}^{2}\right)}
$$

First, efforts are made to suppress error sources by improving hardware and/or alignment methods. Then any residual systematic errors are evaluated offline to the best precision possible i.e. to approach an estimate of the "true beam size". The relative "total $\mathrm{M}$ reduction" $\mathrm{C}_{\text {total }}=\prod_{i} C_{i}$ for a particular $\theta$ mode can be observed by comparing the inconsistency in $\sigma_{y, \text { meas }}$ immediately before and after $\theta$ mode switching. However this $\mathrm{C}_{\text {total }}$ cannot be applied universally to any mode because some systematic errors are mode dependent. Estimating the absolute $\mathrm{C}_{\text {total }}$ requires careful quantitative evaluation of each individual source. For some factors we can only get a worst limit. Nevertheless, the merit of using the Shintake Monitor in beam focusing is that we can observe the relative changes in $\sigma_{\mathrm{y}}$ regardless of how much $M$ is systematically reduced, provided all conditions remain stable. Table 2 summarizes the major individual $\mathrm{M}$ reduction sources explained as following. Further details are found in [9].

Laser polarization and power: The Shintake Monitor optics is designed for linearly S polarized laser (w.r.t. optical component surfaces). Measurement of laser polarization indicate the laser to be very close to purely S linear polarized, with "P contamination" $\mathrm{P}_{\mathrm{p}} / \mathrm{P}_{\mathrm{s}}<1.5 \%$. Scanning the angle of a half lambda $(\lambda / 2)$ plate which rotates the laser's polarization state, determines the optimum setting for maximum $\mathrm{M}$ and best power balance between upper and lower paths. Additionally, the reflective ratio for $\mathrm{S}$ light of the $50 \%$ beam splitter was measured to be $\mathrm{R}_{\mathrm{s}}=50.3 \%$, well within catalog specifications. Altogether, these indicate very little $\mathrm{M}$ reduction due to polarization related issues (see [9] for details). Fringe tilt: Mismatch between the beam and the axis of laser fringes leads to fringe tilt in the form of "pitch" or "roll" in the longitudinal or transverse direction, respectively. The contribution of fringe pitch and roll to beam size depends on longitudinal laser spot size $\left(\sigma_{\text {laser }}\right)$ and horizontal IP e- beam size $\left(\sigma_{\mathrm{x}}\right)$, respectively, and is roughly expressed as:

$$
\sigma_{y}^{2} \rightarrow \sigma_{y}^{2}+\sigma_{\text {laser }}^{2} \cdot \Delta \Phi_{\text {pitch }}^{2} \quad \text { and } \quad \sigma_{y}^{2} \rightarrow \sigma_{y}^{2}+\sigma_{x}^{2} \cdot \Delta \Phi_{\text {roll }}^{2}
$$

Both $\sigma_{\text {laser }}$ and $\sigma_{\mathrm{x}}$ are typically around $10 \mu \mathrm{m}$. For example, a tilt of $5 \mathrm{mrad}$ would cause a nominal $\sigma_{\mathrm{y}}=$ $40 \mathrm{~nm}$ to be over-evaluated by about $25 \mathrm{~nm}$ to $65 \mathrm{~nm}$. Although tilt can be suppressed during setup of the laser optics by careful alignment of each path w.r.t the centers of the focal lenses, the laser may have drifted off by the time we conduct fringe scans. Furthermore, the $\mathrm{e}^{-}$beam itself may be rotated in the transverse plane. Therefore we correct fringe tilt using the $\mathrm{e}^{-}$beam as a reference by conducting the "tilt scan"; $M$ is measured while rotating the laser fringes by adjusting intermediate mirrors in the laser paths. The mirrors settings are then set to yield the maximum M.

Phase jitter (relative position jitter): The jitter in the relative phase $(\Delta \varphi)$, or equivalently relative position $\left(\Delta y=\Delta \varphi /\left(2 \mathrm{k}_{\mathrm{y}}\right)\right)$ between laser fringes and beam is one of the potentially dominant $\mathrm{M}$ reduction factors. Since $\Delta \varphi$ is a convolution between laser and beam, it is difficult to distinguish which is jittering. At present, we lack the means of independent measurement for either. If $\mathrm{e}^{-}$beam jitter is dominant, real beam size growth issues need to be addressed. We anticipate measurement by the (nm) resolution "IPBPM" under commissioning[14] for beam jitter at IP, which likely comes from magnet vibrations or extraction jitters from damping ring[15]. Approximated as Gaussian jitters, $\Delta \varphi$ reduces $\mathrm{M}$ as $\mathrm{M}_{\text {meas }}=\mathrm{M}_{0} \cdot \mathrm{C}_{\Delta \varphi}=\mathrm{M}_{0} \cdot \exp \left(-\Delta \varphi^{2} / 2\right)$. Translated to $\Delta \mathrm{y}$, the impact on $\sigma_{\mathrm{y}, \text { meas }}$ is $\sigma_{\mathrm{y} 0}{ }^{2}+\Delta \mathrm{y}^{2}$. Sec 3.5 provides a detailed explanation of $\Delta \varphi$, including demonstration of a method for deriving $\Delta \varphi$ from fringe scan data, and using the extracted $\Delta \varphi$ to correct the measured $\mathrm{M}$ as $\mathrm{M}_{\text {corr }}=\mathrm{M}_{\text {meas }} / \mathrm{C}_{\Delta \varphi}$.

Phase drifts and jumps: There are also sudden phase jumps and drifts which may be hard to distinguish from jitters. The effect on $\mathrm{M}_{\text {meas }}$ depends on their various time structures. These are also a convolution of laser and beam position. Drift of the initial phase in continuous fringe scans is observed to be occasionally as large as $150 \mathrm{mrad} / \mathrm{min}$ over a linear scale. When drift is detected from sudden decrease in Compton signal intensity, the laser is realigned to beam by scanning mirror actuators. 
Laser position and profile at IP: Laser crossing is maintained well aligned to e beam by scanning and adjusting mirror angles using actuators. We aim for alignment precision better than $10 \%$ of laser spot size. However realistically, the laser profile is somewhat deviated from Gaussian distribution, and fluctuates shot-by-shot. This profile degradation may result in $\mathrm{M}$ reduction since it affects laser profile balance and collision with the beam, and therefore signal intensity.

Table 2: Status of individual non-negligible M reduction factors estimated for $\sigma_{\mathrm{y}}$ measurement at $174{ }^{\circ}$ mode . See main text for details.

\begin{tabular}{|l|ll|}
\hline \multicolumn{1}{|c|}{ M reduction factors } & \multicolumn{1}{c|}{ Status } \\
\hline $\begin{array}{l}\text { Phase jitter / drift } \\
\text { (vertical relative position } \\
\text { jitter) }\end{array}$ & $-\begin{array}{l}\text { Convolution of laser and beam position variation at IP, lack independent } \\
\text { measurement of either. }\end{array}$ \\
\hline $\begin{array}{l}\text { Laser profile and position } \\
\text { alignment }\end{array}$ & $\begin{array}{l}\text { Typically } 0.4-0.75 \mathrm{rad} \text { for } 174^{\circ} \text { mode }(\Delta \mathrm{y}=20-30 \mathrm{~nm}) \text {, smaller for } 30^{\circ} \text { mode. } \\
\text { Simulation studies based on realistic conditions are ongoing }\end{array}$ \\
\hline Fringe tilt & $\begin{array}{l}\text { Position adjusted using mirror actuators } \\
\text { profile balance adjusted by scanning position of focal lenses. } \\
\text { shot-by-shot laser profile fluctuation may cause M reduction }\end{array}$ \\
\hline Polarization & Optimized by "tilt scan" \\
\hline
\end{tabular}

\subsection{Signal Jitter Sources}

Fast signal jitter sources that vary shot-by-shot cannot be resolved by simply increasing statistics. These fluctuate the measured signal energy and consequently degrade efficiency of beam tuning and $\mathrm{M}$ reduction evaluation. Very heavy jitters may even hinder detection of the smaller $\mathrm{M}$. At the low beam charge $\left(\leq 1 \times 10^{9}\right)$ typically used for small $\sigma_{\mathrm{y}}$ measurements, signal jitter is $15-20 \%$ at peaks and bottoms of fringe scans and $35-40 \%$ at "mid-points", w.r.t to $\mathrm{E}_{\text {avg. }}$. This may vary depending on conditions of laser and beam. Individual jitter sources are assessed by improving hardware or offline data selection. The major sources are described below and in [9].

BG Fluctuation: Compton signal energy is measured using a background (BG) subtraction method (see [9]). Beam trajectory instability may fluctuate BG levels. Given a sufficiently high S/N ratio of about 20 , this leads to signal jitters of typically $<5 \%$, which is not problematic.

Compton Photon Statistics: The statistical fluctuation of the measured $\mathrm{E}_{\text {sig }}$ is generally proportional to the inverse of the square root of the number of signal photons $\left(\mathrm{N}_{\text {sig }}\right)$ per bunch, scaling as $1 / \sqrt{N_{\text {sig }}}$. $\mathrm{N}_{\text {sig }}$ is affected by e $\mathrm{e}^{-}$beam intensity, laser power at IP, beam orbit, collimation, and resolution of the CsI detector. Estimation using calibration data is described in Sec. 3.4.

Laser Timing and Intensity: Timing jitters in the $3.4 \mathrm{~ns}$ (rms) laser pulses cause pulse-to-pulse inconsistency in the laser power intercepted by much shorter $16 \mathrm{ps} \mathrm{e}^{-}$beam pulses during collision. Laser timing and beam timing are monitored using the signals from PIN-photodiode and strip-line BPMs, respectively. Precise matching is carried out during beam time by scanning the laser Q-switch timing using digital modules. The laser temporal profile observed using an oscilloscope indicates $<$ $10 \%$ of laser power jitter and 1-3 ns (rms) of laser timing jitter. However there are also finer time structures observed in the profile that could possibly affect collision.

Phase jitter (vertical relative position jitter): Sec. 3.2 introduced phase jitter $(\Delta \varphi)$, or equivalently relative position jitter $(\Delta y)$ as one of the potentially dominant $M$ reduction factors. The signal jitter caused by $\Delta \varphi$ depends on signal energy, $M$, and fringe scan phase as:

$$
\Delta E_{p}=E_{\text {avg }} M \sqrt{\frac{1}{2}\left[1-2 \cos ^{2}(\phi) \exp \left(-\Delta \phi^{2}\right)+\cos (2 \phi) \exp \left(-2 \Delta \phi^{2}\right)\right]}
$$

For example, when measuring a nominal $\sigma_{\mathrm{y}}$ of $50 \mathrm{~nm}\left(\mathrm{M}_{0}=0.5\right.$ for $\left.174^{\circ} \operatorname{mode}\right), \Delta \varphi=550 \operatorname{mrad}(\Delta \mathrm{y}=$ $23 \mathrm{~nm}$ ) causes about $9 \%$ and $24 \%$ jitter at peak and bottom and $24 \%$ at mid-point w.r.t $\mathrm{E}_{\text {avg }}$.

Horizontal Relative Position Jitter: Compton intensity is fluctuated by a convolution of horizontal beam jitter at IP and laser pointing jitter. The latter is inferred using laserwire mode data to be about $5 \%$ of the laser spot size. This is consistent with the laser profile jitters monitored using CCD cameras. 
ICT Monitor Resolution: The measured energy is normalized by the measured e beam intensity. In this way, the impact from beam current jitter is suppressed down to the resolution of the "integrated current transformer (ICT) monitor", which about a few \%.

\subsection{Estimation of signal jitters}

Signal jitter $(\Delta \mathrm{E})$ in a fringe scan is defined as the standard deviation of $\mathrm{N}_{\mathrm{av}}$ (= number of pulses) of energy events (E) at each phase. For analysis and simulation, signal jitter is generally represented by Eq. 8 as a convolution of "vertical jitters" $\Delta \mathrm{E}_{\mathrm{V}}$ (Eq. 9) and "horizontal jitters" caused by phase jitters $\triangle \mathrm{E}_{\mathrm{p}}$ (Eq. 7). Each jitter component is assumed to follow a Gaussian distribution.

$$
\begin{aligned}
\Delta E & =\sqrt{\Delta E_{p}^{2}+\Delta E_{V}^{2}} \\
\Delta E_{V} & =\sqrt{C_{\text {const }}^{2}+\left(C_{\text {stat }} \sqrt{E}\right)^{2}+\left(C_{\text {linear }} E\right)^{2}}
\end{aligned}
$$

$\mathrm{C}_{\text {const }}$ is related to BG fluctuations (see Sec. 3.3). $\mathrm{C}_{\text {linear }}$ is related to laser instabilities (position, angle, timing, and power). $\mathrm{C}_{\text {stat }}$ is related to Compton photon statistics, which depends on properties of detector and collimation. Typically, $\mathrm{C}_{\text {stat }}$ is dominant over $\mathrm{C}_{\text {const }}$ and $\mathrm{C}_{\text {linear }}$.

The following calibration is performed for evaluating linearity and fluctuations related to the response of the CsI detector. Beam bunch charge was changed in steps while recording $\mathrm{E}$ and $\Delta \mathrm{E}$, defined as mean and standard deviation, respectively, of multiple signal energy events measured by the detector. Only the upper laser path was let out to IP, and its position was set to optimize collision with the beam. Fig. 5 left shows sufficient linearity of $\mathrm{E}$ over a wide beam intensity range. Fig. 5 right shows $\Delta \mathrm{E}$ as a function of $\mathrm{E}$, and fitted with Eq. 10 with 3 free parameters $\mathrm{C}_{1}, \mathrm{C}_{2}$, and $\mathrm{C}_{\text {stat }}$.

$$
\Delta E=\sqrt{C_{1}^{2}+\left(C_{s a t} \sqrt{E}\right)^{2}+\left(C_{3} E\right)^{2}}
$$

Here, $\mathrm{C}_{1}$ is mainly from electrical noise of the detector. $\mathrm{C}_{2}$ is mainly from intensity fluctuation of beam and laser. $\mathrm{C}_{\text {stat }}$ is from the statistical fluctuation of the number of Compton photons. The fitted result gives $C_{\text {stat }}=6.2 \cdot \sqrt{\text { unit of } E}$. This emphasizes that detector related fluctuation depends on energy as $C_{\text {stat }} / \sqrt{E}$, which contributes about $17 \%$ to signal jitters (w.r.t. $\mathrm{E}_{\text {avg }}$ ) for low E orresponding to low bunch charge $1 \times 10^{9}$, and about $10 \%$ for a higher E corresponding to low bunch charge $3 \times 10^{9}$ (see Fig. 5 left). This $\mathrm{C}_{\text {stat }}$ from calibration is expected to be consistent as that in Eq. 9 for the same CsI detector condition, and is thus used for $\Delta \varphi$ analysis (in Sec. 3.5) .
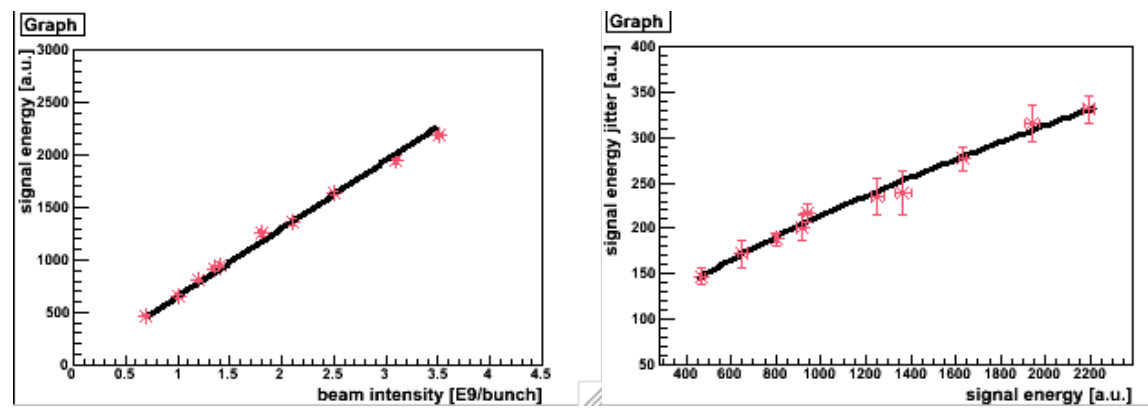

Fig 5 : (left) Demonstration of linearity of the CsI detector by plotting energy measured as a function of beam bunch charge $\left(\mathrm{I}\left[10^{9}\right]\right)$. Fitted result is : E [a.u $]=650^{\prime} \mathrm{I}$ (right) Energy jitter plotted as a function of energy, and fitted with eq(9)

\subsection{Development of a Phase Jitters Extraction Method}

This section describes the method developed for deriving the systematic error due to $\Delta \varphi$. Signal jitter is plotted as a function of $\varphi$, then fitted with a model (Eq. 8) which assumes a convolution of Gaussian distributed components due to vertical jitters and to phase jitters. $\Delta \varphi$ is extracted from fitting by $\chi^{2}$ minimization along with $C_{\text {linear }}$. The fixed parameters are $M, E_{\text {avg }}$, and $\varphi_{0}$ from Eq. 4 , and the realistically estimated values of the other relatively time stable vertical jitter components. Fig 6 shows this method applied to an actual $174^{\circ}$ mode $\mathrm{N}_{\mathrm{av}}=50$ fringe scan in May, 2014. The extracted $\Delta \varphi=0.74+/-0.03 \mathrm{rad}$ corresponds to relative position jitter $\Delta y=31+/-1 \mathrm{~nm}$. In reality, $\Delta \varphi$ is a combination of jitters and drifts, and its effect depends on the time structure of the fringe scan. Typical 
$\Delta \varphi$ in $174^{\circ}$ mode scans in April-June of 2014 ranges as $400-750$ mrad. corresponding to $\Delta y$ of $15-30$ $\mathrm{nm} . \Delta \varphi$ is smaller for $30^{\circ}$ mode, which may be explained by that the shorter path length receives less effect from laser pointing jitter, and that the same e beam jitter leads to less $\Delta \varphi$ for a smaller $\theta$ mode.

Since $\Delta \varphi$ is one of the dominant systematic errors for $\sigma_{\mathrm{y} \text {,meas }}$, analysis precision of $\Delta \varphi$ is demonstrated under a variety of scenarios using simulation. One example is described below.

\section{Simulation using an example of realistic phase jitter and drift}

The phase variation of the laser fringes had been measured by T.Yamanaka in 2009[16] using a previously installed phase monitor. This used a different laser from the current one, and took place during beam off time, thus excludes e' beam jitters. The reproduced version of this data (Fig. 7 left), whose $\mathrm{rms}$ is $0.448 \mathrm{rad}$, was input into fringe scan simulation for $\sigma_{\mathrm{y} 0}=40 \mathrm{~nm} \mathrm{M}_{0}=0.636$ for $174^{\circ}$ mode, along with a particular realistic scenario of a linear phase drift of $80 \mathrm{mrad} / \mathrm{min}$ and a $0.7 \mathrm{rad}$ jump at $\varphi=11 \mathrm{rad}$. Vertical jitter input gives $5 \%, 23 \%$ and $10 \%$ jitters w.r.t. $E_{\text {avg }}$ from $C_{\text {const, }} C_{\text {stat }}$, and $\mathrm{C}_{\text {linear }}$ in Eq. 9 respectively. The aim is to observe the effect on $\mathrm{M}_{\text {meas }}$ from this non-Gaussian fluctuation (" $\Delta \varphi_{\text {in }}$ ") and to demonstrate extraction of $\Delta \varphi\left(\Delta \varphi_{\text {out }}\right)$ and using it to correct $\mathrm{M}_{\text {meas }}$.

Fig 7 right compares these results between $\mathrm{N}_{\mathrm{av}}=10,20,50$, and 100. The systematic effect on $\mathrm{M}_{\text {meas }}$ depends on the time structure of the fluctuation w.r.t. that of the scan, which depends on $\mathrm{N}_{\mathrm{av}}$. The same phase fluctuation $\Delta \varphi_{\text {in }}$ behaves as Gaussian like jitters for a large $N_{\mathrm{av}}$ and a combination of jitters and slower drifts for a small $\mathrm{N}_{\mathrm{av}}$. For a larger $\mathrm{N}_{\mathrm{av}}$, although $\mathrm{M}_{\text {meas }}$ reduction tends to more apparent, it is more suited to the Gaussian $\Delta \varphi$ extraction model, thus yielding slightly better precision in correction of $\mathrm{M}_{\text {meas }}$ as $\mathrm{M}_{\text {corr }}=\mathrm{M}_{\text {meas }} / \mathrm{C}_{\Delta \varphi}$. In Fig 8, the amplitude of the fine $\Delta \varphi$ in Fig. 7 left is multiplied by $\{0$, $0.5,1,1.5,2\}$ times to test the $\mathrm{N}_{\mathrm{av}}=50$ case. Fig 8 left shows $\Delta \varphi_{\text {out }}$ precision to be better than a few $\%$ w.r.t. the amplitude (rms), with exception of the "fake $\Delta \varphi$ " of about $40 \mathrm{mrad}$ at zero amplitude input due to coupling effects from vertical jitters. Fig 8 right shows that regardless of $\Delta \varphi_{\text {in }}$ amplitude, $M_{\text {meas }}$ can be corrected back to nominal with better than a few \% accuracy.

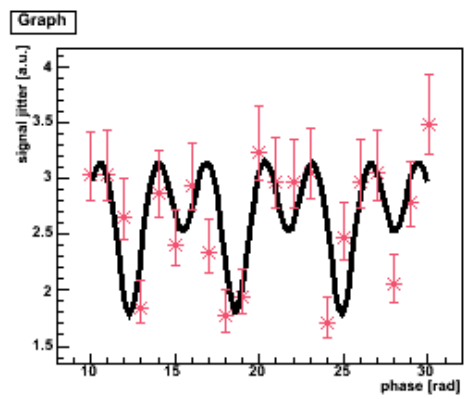

Fig $6: \Delta \varphi$ for an example $174^{\circ}$ mode $\mathrm{N}_{\mathrm{av}}=50$ scan is estimated as $0.74+/-0.03 \mathrm{rad}$, corresponds to $\Delta \mathrm{y}=31+/-1 \mathrm{~nm}$
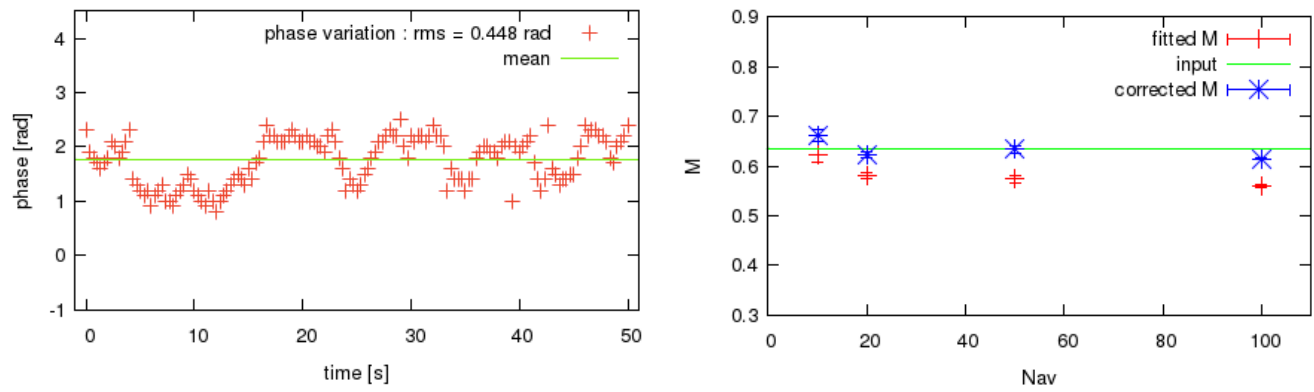

Fig 7: (left) Measured fringe phase variation from 2009 (right) was reproduced for 150 pulses in $3 \mathrm{~Hz}$ intervals and input repeatedly into simulation of $174^{\circ}$ fringe scan assuming $\mathrm{M}_{0}=0.636, \sigma_{\mathrm{y} 0}=40 \mathrm{~nm}$ along with a linear drift of $80 \mathrm{mrad} / \mathrm{min}$, a $0.7 \mathrm{rad}$ jump at $\varphi=11 \mathrm{rad}$, and realistic vertical jitters. The effect on $\mathrm{M}_{\text {meas }}$ and $\mathrm{M}_{\text {corr }}$ (corrected using $\Delta \varphi_{\text {out }}$ ) is shown for $\mathrm{N}_{\mathrm{av}}=10,20,50$, and 100 , as the statistical results of 10 random seeds. 

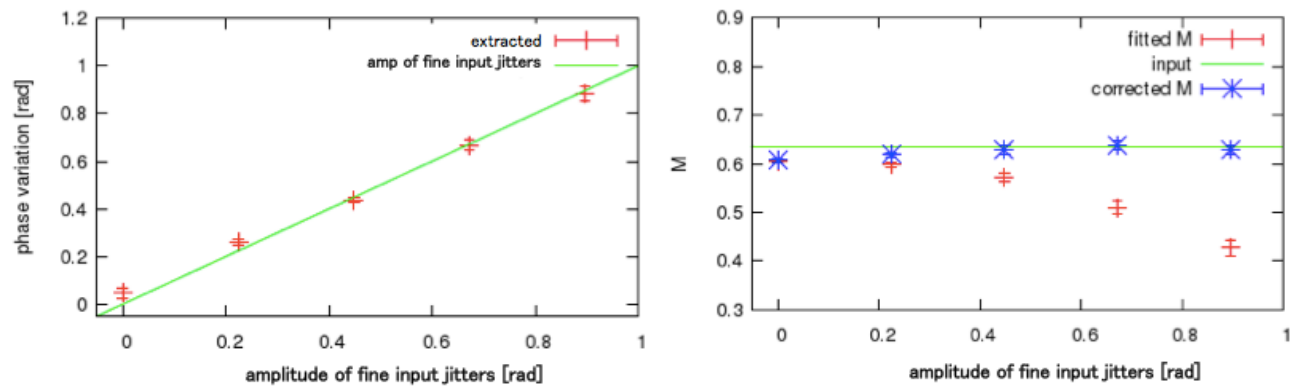

Fig. 8: Simulation using various amplitudes of the "fine" phase variation in Fig 7 left (other inputs, e.g. linear drift and jump are kept same). Results are shown for the $\mathrm{N}_{\mathrm{av}}=50$ case as the statistical results of 10 random seeds. (left) : With exception of 0 amplitude input, a wide range of $\Delta \varphi$ can be extracted (red) with less than a few \% deviation w.r.t. amplitude of the "fine" jitters (green). (right) : the fitted $\mathrm{M}_{\text {meas }}$ can be corrected with better than few $\%$ accuracy back to nominal $\mathrm{M}_{0}=$ 0.636 i.e. there is little residual $\mathrm{M}$ reduction.

\section{Summary and Future Goals}

The Shintake Monitor measures the focused vertical e beam sizes $\left(\sigma_{\mathrm{y}}\right)$ at the IP of ATF2 using laser interference fringes as a target for the beam. It plays an indispensable role in demonstrating a final focus system for realizing high luminosity for ILC. Measurement stability of about 5\% enables effective beam tuning and progress moving towards achieving the ATF2 goal $\sigma_{\mathrm{y}}$ of $37 \mathrm{~nm}$. Hardware improvements to suppress signal jitters and drifts, as well as detailed study of various systematic errors have been ongoing in order to enable precise beam size evaluation.

\section{Acknowledgements}

We would like to acknowledge the members of the ATF collaboration, in particular of the ATF2 beam tuning group for the beam time progress reported in this paper. The first author, J.Yan, would like to thank Prof. Phillip Bambade of LAL who had first motivated her to commence the study of phase jitters. J. Yan would also like to thank the Japan Society For The Promotion of Science for financial support throughout her PhD. research at the Graduate School of University of Tokyo.

\section{References}

[1] ILC Technical Design Report, 12 June 2013

[2] P.Raimondi,A.Seryi,PRL,86,3779(2001)

[3] ATF2 group, ATF2 Proposal, KEK Report 2005-2

[4] T. Shintake, "Proposal of Nanometer beam size monitor for e+e- colliders", Nucl. Instrum. and Meth. In Phys, Research, A311 (1992) 453-464

[5] T. Suehara et al, : "A Nanometer Beam size Monitor for ATF2" in Nucl. Instrum. and Meth. In Phys, Research A 616 (2010) $1-8$

[6] P.G.Tenebaum, "Expanded Studies of Linear Collider Final Focus Studies at the Final Focus Test Beam", SLAC Report-95-475 (1995).

[7] Y. Yamaguchi, Master thesis at Graduate School of Science, The University of Tokyo (2010)

[8] J. Yan, Master thesis at Graduate School of Science, The University of Tokyo (2011)

[9] J.Yan et al: Measurement of Nanometer Electron Beam Sizes with Laser Interference using Shinrtake Monitor in Nucl. Instrum. and Meth. In Phys, Research A740 (2014) 131-137

[10] T.Okugi et al., Linear and second order optics corrections for the KEK Accelerator Test Facility final focus beam line, PHYSICAL REVIEW SPECIAL TOPICS - ACCELERATORS AND BEAMS 17, 023501 (2014)

[11] The ATF Collaboration: "Experimental validation of a novel compact focusing scheme for future energy frontier linear lepton colliders", Phys. Rev. Lett. 112, 034802

[12] K. Kubo and the ATF2 Collaboration: Towards International Linear Collider: Experiments at ATF2, Presentation slides and proceeding for the 5th International Particle Accelerator Conference (IPAC14), 2014

[13] K. Kubo et al : Wakefield Issues for the Linear Colliders, ICFA Beam dynamics Newsletter. No. 61. (2013)

[14] S. Jang et al: The High Position Resolution Cavity BPM Development and Measurement for ILC Final Focus System, proceeding for IPAC14

[15] J. Pfingstner et al. Localisation of beam offset jitter sources at ATF2. Proceeding for IPAC14

[16] Y. Yamanaka, Master thesis at Graduate School of Science, The University of Tokyo (2009) 


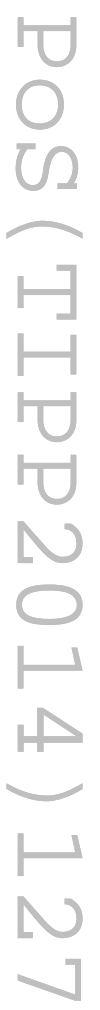

